\section{Las tecnologías de la información y la comunicación en el soporte social dirigido a cuidadores familiares de personas con enfermedad crónica ${ }^{\dagger}$}

\author{
Lucy Barrera Ortiz * \\ Ángela María Ceballos Villa** \\ Susana Jimena Mora Arellano***
}

ISSN 1794-9831

E-ISSN 2322-7028

Vol. 12 No. 1

Ene - Jun 2015

Cúcuta, Colombia

Recibido:

12 de Noviembre

de 2014

Aprobado:

25 de Mayo

de 2015

* Enfermera

Magister en

Enfermería con

énfasis en Salud

Familiar. Profesora

Titular. Universidad

Nacional de

Colombia. Correo

electrónico:

lbarrerao@unal.

edu.co

** Enfermera.

Universidad

Nacional de

Colombia.

Correo electrónico:

amceballosvi@unal.

edu.co

*** Enfermera.

Universidad

Nacional de

Colombia. Correo

electrónico:

sjmoraa@unal.

edu.co

**** Descriptores

en Ciencias de la

Salud (DeCS), en

la página http://

decs.bvs.br/E/

homepagee.htm

de la Biblioteca

virtual en salud del proyecto BIREME, de la Organización

Mundial de la Salud y de la Organización

Panamericana de

Salud.

Para citar este artículo / To reference this article / Para citar este artigo

Barrera-Ortiz L, Ceballos-Villa AM, Mora-Arellano SJ. Las tecnologías de la información y la comunicación en el soporte social dirigido a cuidadores familiares de personas con enfermedad crónica. Rev. cienc. cuidad. 2015; 12(1): 53-63.

† Trabajo de Grado en Pasantía financiado por el Programa para la Disminución de la Carga de la Enfermedad Crónica en Colombia. Colciencias. 2011-2014. 


\section{The information and communications technologies in the social support aimed at family caregivers of people with chronic illness}

\section{ABSTRACT}

Objective: to identify and respond to the needs of family caregivers of people with chronic illness using information and communication technologies. Materials and Methods: qualitative study, it was carried out during the first semester of 2014, under the action research method that allowed simultaneously meet care problems experienced by caregivers of people with chronic illness and generate a response to the needs identified by using a page Web. Results: the family caregivers of people with chronic disease identified as their most sensitive problems of impotence, fear, hopelessness and isolation. Because of this, it was created a frequently asked questions section available via web page thereby seeking to generate a mechanism of social support. Conclusions: the identification of the needs of families of people with chronic disease caregivers allowed to give a specific answer supported by the information and communication technologies is offered as a source of permanent access to satisfy their concerns.

KEYWORDS: social support, health communication, caregivers, webcasts. 


\section{A tecnologia da informação e das comunicações em apoio social destinada a cuidadores familiares de pessoas com doenças crónicas}

\section{RESUMO}

Objetivo: identificar e responder às necessidades de cuidadores familiares de pessoas com doenças crónicas, utilizando tecnologias de informação e comunicação. Materiais e Métodos: estudo qualitativo, realizado durante o primeiro semestre de 2014, sob o método de pesquisa-ação que permitiu simultaneamente atender problemas de cuidados vivenciados por cuidadores de pessoas com doença crônica e gerar uma resposta às necessidades identificadas usando uma página Rede. Resultados: os cuidadores familiares de pessoas com doença crônica identificados como seus problemas mais sensíveis da impotência, medo, desesperança e isolamento. A partir dele uma seção perguntas frequentes disponível através da página web gerado buscando gerar um mecanismo de apoio social. Conclusões. A identificação das necessidades das famílias de pessoas com cuidadores de doenças crônicas autorizados a dar uma resposta específica apoiada pela tecnologia da informação e das comunicações é oferecido como uma fonte de acesso permanente para satisfazer as suas preocupações.

PALAVRAS-CHAVE: apoio social, comunicação em saúde, cuidadores, webcasts. 


\section{INTRODUCCIÓN}

Ene - Jun 2015 Cúcuta, Colombia
$\mathrm{L}$ as enfermedades crónicas son de larga duración, por lo general de progresión lenta y se caracterizan por el significativo impacto que generan tanto en quienes las padecen como en sus grupos familiares, especialmente en sus cuidadores familiares (1-2). En muchas ocasiones, los familiares deben asumir el cuidado de sus seres queridos, tarea para la cual se requiere conocimiento y apoyo especializado, fundamental para afrontar la experiencia, pero que no siempre resulta disponible (3-4). Las consecuencias de carga, depresión, alteración en la calidad de vida y aumento de la morbilidad en estos cuidadores familiares, han sido ampliamente documentadas (5-10).

El soporte social es un apoyo percibido por el cuidador para afrontar la experiencia de cuidar a su familiar en situación de enfermedad crónica, ha sido descrito por Hilbert en 5 categorías: 1) Interacción personal o apoyo de tipo emocional para el afrontamiento adecuado en diversas situaciones de enfermad crónica; 2) Guía o ayuda que percibe el cuidador con educación e información; 3) Retroalimentación al sentir que quienes les brindan apoyo están de acuerdo con ellos y apoyan sus pensamientos y acciones; 4) Ayuda tangible o material, en tareas físicas y labores desempeñados por el rol del cuidador, y 5) Interacción social o apoyo para permitir que los cuidadores rescaten sus relaciones con otros grupos, familias e instituciones que respondan a sus necesidades (11).

Una revisión sistemática sobre el uso de las tecnologías de la información y la comunicación (TIC) como mecanismo para apoyar a los cuidadores familiares de personas con enfermedad crónica señaló que las TIC, en particular el teléfono y el internet, se asocian con un efecto protector de la salud y del vínculo afectivo y se recomienda incorporarlas en el contexto colombiano y en la práctica de enfermería a través de proyectos de investigación (12).

Estas tecnologías de información y comunicación se han identificado como "estrategias para conformar redes de apoyo y brindar un sistema de soporte social con mayor seguimiento, que integra conexiones a través de medios de comunicación y soporte en línea, facilitando interacciones permanentes entre los pacientes con enfermedad crónica, sus cuidadores familiares y el sistema de salud" (13).
Se ha señalado que la internet es un medio social y puede ser utilizado para el intercambio de información de apoyo que permite comunicarse con las personas que no se encuentran tradicionalmente en el círculo de relaciones sociales, logrando, de forma adicional, que se disminuya la ansiedad que representa estar frente a frente (14).

Tres ejemplos de utilización de las TIC en el área de la salud ratifican los hallazgos mencionados en este estudio. El programa SOS Telemedicina para Venezuela que, de manera gratuita, ofrece servicios de teleconsulta y telediagnóstico, los cuales son entregados por profesores calificados de diferentes especialidades de la salud; el Proyecto de Telemedicina de la Fundación Cardiovascular de Colombia, que busca generar servicios de salud equitativos y accesibles especialmente para quienes viven en zonas apartadas, y las ofertas específicas para cuidadores con empleo de TIC del Programa Cuidando a los Cuidadores de la Universidad Nacional de Colombia (15-17).

\section{OBJETIVOS}

\section{Objetivo general}

Identificar y responder a las necesidades referidas por los cuidadores familiares de personas con enfermedad crónica, mediante el uso de TIC.

\section{Objetivos específicos}

- Determinar la percepción de los cuidadores familiares de personas con enfermedad crónica en fase avanzada respecto a la información que reciben.

- Generar una estrategia de intervención con el uso de las TIC a partir de las necesidades referidas por los cuidadores.

\section{MATERIALES Y MÉTODOS}

Este es un estudio cualitativo que empleó el método de investigación acción y fue realizado durante el primer semestre de 2014, en el marco del Programa de Investigación para la Disminución de la Carga de la Enfermedad Crónica en Colombia. El método de investigación acción, como su nombre lo indica, da la posibilidad a los investigadores de generar conocimiento a la vez que les permite actuar en respuesta a la realidad social. El método se compone 
de tres fases denominadas descongelamiento, movimiento y recongelamiento (18).

En este caso, se inició reconociendo los problemas del cuidado de acuerdo con la realidad de los cuidadores de personas con enfermedad crónica vinculadas al Programa Cuidando a los Cuidadores (19).

Se generó a partir de ello un proceso de respuesta a las necesidades identificadas mediante el uso de una página Web y se abrió al público para que la misma fuese empleada por estos y otros cuidadores que puedieran beneficiarse de este desarrollo. Se utilizaron como técnicas de recolección de información la entrevista semiestructurada y las narrativas referidas por los participantes a través de la página web.

La muestra estuvo integrada por 12 cuidadores familiares de personas con enfermedad crónica que se encontraban en fase final o avanzada, vinculados a programas o instituciones de cuidado, con acceso, uso y conocimiento del computador y el internet. Se consideró un muestreo intencional a conveniencia orientado por los criterios de saturación teórica de la información.

Se cumplieron los siguientes pasos:

1. Reconocer la insatisfacción con el tipo de información disponible a los cuidadores familiares.

2. Identificar cuál era su percepción del problema.

3. Puntualizar la forma como el problema manifestado podría ser resuelto o minimizado mediante la acción.
4. Formular hipótesis para seleccionar la más adecuada; desarrollar la acción propuesta $\mathrm{y}$, a partir de ello, evaluar sus efectos.

5. Generalizar la utilización de los productos desarrollados (18).

El estudio fue avalado por el Comité de ética institucional y mantiene todos los requisitos exigidos por la Resolución No. 8430 de 1993 (20), por la cual se establecen las normas científicas, técnicas y administrativas para la investigación en salud en Colombia. Se acogió la política ambiental de uso responsable de recursos (21).

\section{RESULTADOS Y DISCUSIÓN}

\section{Reconocimiento de la insatisfacción}

Se identificó que los cuidadores familiares tenían varias necesidades de información sobre aspectos relacionados con su vida cotidiana que les resultaba indispensable resolver para ejercer el rol de cuidadores. Esta información se obtuvo por medio de entrevistas semiestructuradas a partir de la vinculación a 3 talleres.

\section{Percepción del problema}

Desde las narrativas se determinó, de forma inductiva, la percepción que los cuidadores tienen sobre el problema identificado. Se refleja en cuatro temas que se constituyen, a la vez, en necesidades de cuidado: impotencia, temor, desesperanza y aislamiento. A continuación se ilustra la forma como esta identificación se desarrolló (ver tabla 1). 
Tabla 1. Ejemplo de identificación de necesidades de los cuidadores familiares de personas con enfermedad crónica.

\begin{tabular}{|c|c|}
\hline EXPERIENCIAS & NECESIDADES IDENTIFICADAS \\
\hline $\begin{array}{l}\text { "iCuando veo a mi madre tan fuera de esta realidad, tan desubicada, } \\
\text { después de ser una persona tan activa y tan independiente, no sé qué hacer, } \\
\text { me desespera no poder ayudarla!" } \\
\text { "Yo no sé muchas cosas de su enfermedad, no sé qué hacer si ella se pone } \\
\text { mal" } \\
\text { "Cuando la veo con dolor, con las piernitas inflamadas, siento que no } \\
\text { puedo hacer nada en casa y que tengo que salir corriendo para el hospital, } \\
\text { pero esto es mucho más difícil" }\end{array}$ & IMPOTENCIA \\
\hline $\begin{array}{l}\text { "Siempre que salgo de la casa, a hacer alguna diligencia, me da miedo } \\
\text { dejar solo a mi familiar y que le pase algo, que precisamente yo no esté } \\
\text { para ayudarlo" } \\
\text { "Yo entiendo que la enfermedad crónica avanza pero no quiero pensar en } \\
\text { cómo ésta la afectará ni física ni psicológicamente" }\end{array}$ & TEMOR \\
\hline $\begin{array}{l}\text { "Es muy difícil hacerse a la idea de que ya no hay tratamiento médico que } \\
\text { cure la enfermedad" } \\
\text { "No sé con quien hablar, muchas personas no me entienden, sólo le pido a } \\
\text { Dios paciencia y que me ilumine para saber cómo apoyar a mi familiar" }\end{array}$ & DESESPERANZA \\
\hline $\begin{array}{l}\text { "Cuando empecé a cuidar a mi familiar sentí que me aleje de muchos } \\
\text { amigos, de muchas personas cercanas, incluso de mis otros familiares", } \\
\text { "Ahora casi no tengo tiempo para salir, para compartir con personas } \\
\text { diferentes, siempre tengo que permanecer en la casa al pendiente de mi } \\
\text { familiar" }\end{array}$ & AISLAMIENTO \\
\hline
\end{tabular}

Fuente: Datos del estudio.

\section{Desarrollo de acciones}

A partir de la identificación de la percepción del problema por parte de los cuidadores de personas con enfermedad crónica, se identificó que cada una de las inquietudes manifestadas con mayor frecuencia por ellos podía ser convertida en una pregunta para buscar una solución en la que, además de los cuidadores, intervinieran los expertos o profesionales que orientan los talleres. Seguidamente se ilustra, con base en el mismo ejemplo anterior, la forma como se identifica la manera en que los problemas que perciben los cuidadores familiares se transforman en preguntas para buscar atenderlos (ver tabla 2). 
Tabla 2. Ejemplo de la forma como el problema manifestado por los cuidadores familiares se convierte en preguntas para buscar ser atendido.

\begin{tabular}{|c|c|}
\hline NECESIDADES & PREGUNTAS CONCRETAS PARA RESOLVER EL PROBLEMA MANIFESTADO \\
\hline IMPOTENCIA & $\begin{array}{l}\text { ¿Puedo obtener atención domiciliaria para mi familiar por parte de la EPS? } \\
\text { ¿Puedo encontrar más información sobre medicamentos con el uso de TIC? } \\
\text { ¿Cómo puedo identificar los problemas y riesgos del paciente y del cuidador? } \\
\text { ¿Qué son los primeros auxilios y por qué debo conocerlos? } \\
\text { ¿Puedo encontrar apoyo instrumental por medio de las TIC? } \\
\text { ¿Qué ventajas tiene la consulta en cuidados paliativos? } \\
\text { ¿Qué debo hacer en caso de que mi familiar presente dolor? } \\
\text { ¿Cómo puedo ayudar a mi familiar cuando no desea comer? } \\
\text { ¿Qué puedo hacer cuando mi familiar presenta vómito? } \\
\text { ¿Qué puedo hacer en caso de que mi familiar presente estreñimiento? } \\
\text { ¿Qué debo hacer cuando mi familiar presenta diarrea? } \\
\text { ¿Por qué debo cuidar la piel de mi familiar y cómo debo hacerlo? } \\
\text { ¿Cómo puedo ayudar a la persona que cuido cuando presenta cambios emocionales? } \\
\text { ¿Qué puedo hacer si la persona a la que cuido no quiere ir al médico o no quiere tomar } \\
\text { los medicamentos? }\end{array}$ \\
\hline TEMOR & $\begin{array}{l}\text { ¿Cómo puedo identificar la parte positiva de la experiencia de cuidar a otro? } \\
\text { ¿Cómo puedo solicitar el servicio de ambulancia en una urgencia? } \\
\text { ¿Qué debo hacer si necesito información acerca de la enfermedad de mi familiar? } \\
\text { ¿Qué tan importante es la familia para el paciente que se encuentra en cuidado paliativo? } \\
\text { ¿Cómo llevo a cabo el proceso de aceptación de la enfermedad de mi familiar? } \\
\text { ¿Cómo afrontar un proceso de duelo? }\end{array}$ \\
\hline DESESPERANZA & $\begin{array}{l}\text { ¿Cuándo debo buscar apoyo? } \\
\text { ¿Qué debo hacer para lograr apoyo emocional? } \\
\text { ¿Qué puedo hacer cuando quiero desahogarme? } \\
\text { ¿Dónde puedo encontrar apoyo para fortalecer mi espiritualidad? } \\
\text { ¿De quién puedo recibir apoyo psicológico y emocional? } \\
\text { ¿Cómo le brindo acompañamiento a la persona que cuido? } \\
\text { ¿Cómo obtengo apoyo espiritual para mí y para la persona que se encuentra bajo mi } \\
\text { cuidado? }\end{array}$ \\
\hline AISLAMIENTO & $\begin{array}{l}\text { ¿Qué debería ser lo más importante para mí cuando cuido de alguien más? } \\
\text { ¿Puedo hacer parte de un grupo o asociación para familiares y/o pacientes con } \\
\text { enfermedad crónica? } \\
\text { ¿Si estoy en Bogotá, que opciones de tipo recreativo/deportivo puedo encontrar? } \\
\text { ¿Cómo puedo hacer parte de mi comunidad y así ampliar mis redes sociales? }\end{array}$ \\
\hline
\end{tabular}

Fuente: Datos del estudio.

\section{Formulación de hipótesis y selección de la más adecuada}

Las preguntas formuladas son consideradas como válidas para atender cada una de las necesidades de los cuidadores, a través de la página Web. Para el estudio se asume como válida e importante cada una de sus quejas que pretende ser resuelta con y para los mismos cuidadores. Además, esta fase permitió la validación de los hallazgos de las etapas previas con los cuidadores, quienes indicaron que la información descrita y las preguntas formuladas eran reflejo de su pensar y sentir respecto a la situación que estaban afrontando.

\section{Desarrollo de la acción propuesta y evaluación}

A partir de la formulación de las preguntas, se definieron las respuestas que inicialmente se recogieron del mismo grupo de cuidadores y fueron complementadas y evaluadas con el apoyo de profesionales expertos en el tema de cuidado al cuidador, quienes, a su vez, tienen experiencia propia como cuidadores. Se buscó que las respuestas, además de ser comprensibles utilizando el lenguaje que emplean los cuidadores, generaran alternativas favorables para resolver sus situaciones de cuidado o para minimizar los efectos negativos. A continuación se ejemplifican las preguntas o acciones generadas (ver tabla 3). 
Tabla 3: Preguntas frecuentes y respuestas de los cuidadores familiares de personas con enfermedad crónica.

PREGUNTAS FRECUENTES

¿Puedo obtener atención domiciliaria por parte de la EPS para mi familiar?

¿Cómo puedo identificar la parte positiva de la experiencia de cuidar a otro?

¿Cuándo debo buscar apoyo?

\section{RESPUESTAS}

$\mathrm{Si}$, la atención domiciliaria se brinda en los casos que se considere necesario por el profesional médico tratante, quien se encarga de evaluar las limitaciones para el autocuidado o el grado de dependencia del paciente para la realización de actividades básicas.

El ser cuidador de un paciente con una enfermedad crónica trae consigo muchas experiencias de todo tipo. Es importante identificar las situaciones que se presentan, muchas veces fortalecemos aspectos espirituales, relaciones personales con nuestra familia o con la persona a la que cuidamos; esto permite que, con el tiempo y la experiencia obtenida, se puedan transmitir conocimientos a otros en forma de ayuda, lo cual genera gran satisfacción personal.

Podemos hacer una lista de cosas positivas que le han pasado o sentido durante el tiempo que cuida a su familiar. Por ejemplo, que mi familia se ha unido más, que he aprendido a conocer realmente a mi familiar, a compartir momentos con esta persona, que he fortalecido aspectos espirituales, conocer características de la enfermedad de mi familiar, tener una satisfacción personal al observar cambios en la persona que cuidamos, al ver mejoría o disminuir estados de ansiedad o de incertidumbre.

Debe buscar apoyo cuando sienta ansiedad o si siente que su vida esta desorganizada, cuando experimente una pérdida o emociones como incertidumbre, amenaza, tristeza, angustia, si se siente cansado (a), agotado (a), con mucho estrés o su carácter cambia constantemente y se molesta con otras personas fácilmente.

$\mathrm{Si}$, puede hacer parte de estos grupos o asociaciones. Para ello es necesario obtener información en su centro de salud, directorio telefónico o páginas de internet, ya que existen asociaciones para pacientes con una enfermedad específica y otras que son más genéricas. Algunos ejemplos de estas asociaciones son:

1. Asociación Colombiana de personas con esquizofrenia y sus familias (http://www.acpef.com/)

¿Puedo hacer parte de un grupo o asociación para familiares y/o pacientes con enfermedad crónica?

2. Asociación Colombiana de pacientes con enfermedades de depósito lisosomal (http://www.acopel.org.co/index.php?option...id.)

3. Asociación Colombiana de Diabetes http://www.asodiabetes.org/

4. Fundación Acción Alzheimer Colombia http://www.alzheimercolombia.org/

5. Fundación Liga Central contra la Epilepsia http://www.epilepsia.org/

(Continúa)

Fuente: Datos del estudio.

\section{Generalizar la utilización de los productos desarrollados}

Por último, para generalizar la utilización de productos, se subieron a una Página Web estos enunciados, en forma de preguntas frecuentes de los cuidadores, para que desde allí pudieran ser consultadas por las personas que viven esta experiencia, retroalimentadas $\mathrm{y}$ continuamente revisadas para dar respuesta a nuevas inquietudes.

\section{CONCLUSIONES}

Este estudio de naturaleza cualitativa, con el método de investigación acción, permitió conocer los problemas del cuidado que viven los cuidadores familiares, desde su propia perspectiva, y construir una respuesta a las necesidades identificadas mediante el uso de una página Web.

Dentro de los problemas identificados por los cuidadores están la impotencia, el temor, la 
desesperanza y el aislamiento. Conocerlos permitió generar una respuesta concreta como un mecanismo de apoyo que permita dar soporte social. Así, puede observarse cómo se abren nuevas estrategias de trabajo, canales y entornos de comunicación desde la promoción de la salud, la prevención y la restauración de la enfermedad, en las que las TIC, en este caso la página Web, se presentan como mecanismo de apoyo para la atención de esta población.

Es necesario diseñar e implementar más estrategias que hagan uso efectivo de las TIC en el sector salud en Colombia, las cuales estén dirigidas tanto a los pacientes como a sus cuidadores familiares, teniendo en cuenta el contexto y la accesibilidad de la población a la tecnología. Para esto, un aspecto fundamental es el conocimiento de todos los trabajadores de la salud acerca de la importancia y la variedad de herramientas que tienen a su alcance para brindar una atención optima, oportuna y accesible.
ISSN 1794-9831

E-ISSN 2322-7028

Vol. 12 No. 1

Ene - Jun 2015

Cúcuta, Colombia 


\section{REFERENCIAS BIBLIOGRÁFICAS}

1. Organización Mundial de la Salud. Enfermedades crónicas. [Internet]. 2014 [consultado 12 de noviembre de 2014]. Disponible en: http://www.who.int/topics/chronic_diseases/es/

2. Ledón L. Enfermedades crónicas y vida cotidiana. Rev Cubana Salud Pública [Internet]. 2011 [consultado 12 de noviembre de 2014]; 37(4):488-499. Disponible en: http://bvs.sld.cu/revistas/spu/vol_37_04_11/spu13411.htm

3. Vega O, González D. Apoyo social: elemento clave en el afrontamiento de la enfermēedad crónica. Enferm. Glob [Internet]. 2009 Jun [consultado 12 de junio de 2014]; 16. Disponible en: http://scielo.isciii.es/scielo. php?script $=$ sci_arttext\&pid=S1695-61412009000200021\&lng=es

4. Barrera L, Pinto N, Sánchez B. Evaluación de un programa para fortalecer a los cuidadores familiares de enfermos crónicos. Revista de Salud Pública. 2006; 8(1): 141- 152

5. Jofré V, Sanhueza O. Evaluación de la sobrecarga de cuidadoras/es informales. Cienc. enferm. 2010; 16( ): 111120.

6. Flores E, Rivas E, Seguel F. Nivel de Sobrecarga en el desempeño del rol del cuidador familiar de adulto mayor con dependencia severa. Cienc. enferm. [Internet]. 2012, Abr [consultado 2 de julio de 2014]; 18(1):29-41. Disponible en: http:/www.scielo.cl/scielo.php?script=sci_arttext\&pid=S0717-95532012000100004\&lng=es http://dx.doi.org/10.4067/S0717-95532012000100004

7. Arechabala M, Catoni M, Palma E, Barrios S. Depresión y autopercepción de la carga del cuidado en pacientes en hemodiálisis y sus cuidadores. Rev Panam Salud Públic [Internet]. 2011 [consultado 2 de julio de 2014]; 30(1):74-79. Disponible en: http://www.scielosp.org/scielo.php?script=sci_arttext\&pid=S102049892011000700011\&lng=en. http://dx.doi.org/10.1590/S1020-49892011000700011

8. López M, Orueta R, Gómez S, Sánchez A, Carmona J, Alonso F. El rol de Cuidador de personas dependientes y sus repercusiones sobre su calidad de vida y su salud. Rev Clin Med Fam [Internet]. 2009 [consultado 2 de julio de 2014]; 2(7):332-339. Disponible en: http://scielo.isciii.es/scielo.php?script=sci_arttext\&pid=S1699695X2009000200004\&lng=es

9. Roca M, Úbeda I, Fuente I, Gallego C, López R, Pont A. et al. Impacto del hecho de cuidar en la salud de los cuidadores familiares. Aten Primaria. 2000; 26 (4): 217-23.

10. Zabalegui A, Juando C, Izquierdo M, González A, Gallart A, Díaz M. et al. Recursos y consecuencias de cuidar a las personas mayores de 65 años: una revisión sistemática. Gerokomos. 2004; 5 (4): 199-208.

11. Hilbert GA. Social support in chronic illness. En: Strickland OL, Waltz CF. Measurement of nursing outcomes: Measuring client self-care and coping skills. Vol. 4. New York: Springer; 1990. p. 79-96.

12. Cárdenas D, Melenge B, Pinilla J, Carrillo G, Chaparro L. Soporte social con el uso de las TIC para cuidadores de personas con enfermedad crónica: un estado del arte. Aquichán. 2010; 10(3): 204-213.

13. Barrera L, Pinto N, Sánchez B. Evaluación de un programa para fortalecer a los cuidadores familiares de enfermos crónicos. Revista de Salud Pública. 2006; 8 (2):141-152.

14. Matthew S, Larose R. Alt. support: modeling social support online. Computers in Human Behavior. 2005; 21: 977992.

15. Comisión Económica para América Latina y el Caribe. Salud y TIC. [Internet]. 2010 [consultado 12 de noviembre de 2014]. Disponible en: http://www.eclac.cl/socinfo/noticias/paginas/3/44733/newsletter12.pdf

16. Corporación Colombia Digital. Apple reconoce proyecto colombiano de telemedicina. [Internet]. 2013 [consultado 12 de noviembre de 2014]. Disponible en: http://www.colombiadigital.net/actualidad/nacional/ item/4839-apple-reconoce-proyecto-colombiano-de-telemedicina.html

17. Barrera L, Carrillo Gloria, Chaparro L, Pinto N, Sánchez B. Soporte social con el uso de TIC's para cuidadores familiares de personas con enfermedad crónica. Rev. salud pública [Internet]. 2011 [consultado 2 de julio de 2014]; 13(3): 446-457. Disponible en: http://www.scielosp.org/scielo.php?script=sci_arttext\&pid=S0124$00642011000300007 \& \operatorname{lng}=\mathrm{en}$

18. Vidal M, Rivera N. Investigación-acción. Educ Med Super [Internet]. [consultado 2 de julio de 2014]; 21(4). Disponible en: http://scielo.sld.cu/scielo.php?script=sci_arttext\&pid=S0864-21412007000400012\&lng=es

19. Barrera L, Pinto N, Sánchez B. «Cuidando a los Cuidadores»: Un programa de apoyo a familiares de personas con enfermedad crónica. Index Enferm [Internet]. 2006 [consultado 2 de julio de 2014]; 15(52-53):54-58. Disponible en: http://scielo.isciii.es/scielo.php?script=sci_arttext\&pid=S1132-12962006000100012\&lng=es

20. República de Colombia. Ministerio de Salud. Resolución No. 008430, octubre 4, por la cual se establecen las normas científicas, técnicas y administrativas para la investigación en salud. Bogotá: Ministerio de Salud; 1993.

21. Universidad Nacional de Colombia. Consejo Superior Universitario de la Universidad Nacional de Colombia. Acuerdo 16 de 2011, octubre18, por el cual se establece la Política Ambiental de la Universidad Nacional de Colombia. Bogotá: Universidad Nacional de Colombia; 2011. 


\section{BIBLIOGRAFÍA}

Klemm P, Bunnell D, Cullen M, Soneji R, Gibbons P, Holecek A. Online Cancer Support Groups: A Review of the Research Literature. The Nurse Practitioner. 2003; 21(3):136-142.

Klemm P, Wheeler E. Cancer Caregivers Online: Hope, emotional roller coaster, and physical/emotional/ psychological responses. CIN: Computers, Informatics, Nursing. 2005; 23 (1): 38-45.

Mason B, Harrison B. Telephone Interventions for Family Caregivers of Patients with Dementia What Are Best Nursing Practices? Feature. 2008; 22 (6): 348-354.

Pierce L, Steiner F, Govoni A. In-home Online Support for Caregivers of Survivors of Stroke: A Feasibility Study. CIN: Computers, Informatics, Nursing. 2002; 20 (4): 157-164. 